\title{
Efficacy of combination of Viola odorata, Rosa damascena and Coriandrum sativum in prevention of migraine attacks: a randomized, double blind, placebo- controlled clinical trial
}

Mohadese Kamali ${ }^{1,2}$, Rostam Seifadini ${ }^{3}$, Hoda Kamali $^{3}$, Mitra Mehrabani ${ }^{4}$, Yunes Jahani ${ }^{5,6}$, Haleh Tajadini ${ }^{2,7}$

${ }^{1}$ MD, Persian Medicine Student, Neurology Research Center, Kerman University of Medical Sciences, Kerman, Iran

${ }^{2}$ Department of Persian Medicine, School of Persian Medicine, Kerman University of Medical Sciences, Kerman, Iran

${ }^{3}$ MD, Neurologist, Assistant Professor, Neurology Research Center, Kerman University of Medical Sciences, Kerman, Iran

${ }^{4}$ Pharmacognosist, Professor, Department of Pharmacognosy, Herbal and Traditional Medicines Research Center, Kerman University of Medical Sciences, Kerman, Iran

${ }^{5} \mathrm{PhD}$ of Biostatistics, Assistant Professor, Modeling in Health Research Center, Institute for Futures Studies in Health, Kerman University of Medical Sciences, Kerman, Iran

${ }^{6}$ Department of Biostatistics and Epidemiology, School of Public Health, Kerman University of Medical Sciences, Kerman, Iran

${ }^{7} \mathrm{MD}, \mathrm{PhD}$ of Persian Medicine, Assistant Professor, Neurology Research Center, Kerman University of Medical Sciences, Kerman, Iran

Type of article: Original

\begin{abstract}
Background: Migraine is the second most common type of headache after tension headaches. In Iranian traditional medicine several herbal drugs are used for the treatment of headache. Including, a product of Iranian traditional medicine, a combination of Viola odorata L. flowers, Rosa damascena L. flowers and Coriandrum sativum L. fruits.

Objective: To determine the effectiveness of a combination of Viola odorata flowers, Rosa damascene flowers and Coriandrum sativum fruits on severity, duration and frequency of migraine headaches.

Methods: This randomized, double blind, placebo-controlled clinical trial was performed on 88 patients who had migraine and visited Besat Neurology Clinic No. 4 at Kerman University of Medical Sciences, Kerman, Iran, from September 2016 to march 2017. Patients were randomly divided into the intervention $(n=44)$ or placebo group $(\mathrm{n}=44)$. The intervention group received a product of Iranian traditional medicine, a combination of Viola odorata L. flowers, Rosa damascena L. flowers and Coriandrum sativum L. fruits in $500 \mathrm{mg}$ capsules three times a day and propranolol $20 \mathrm{mg}$ tablet twice a day, and the control group received placebo capsules $(500 \mathrm{mg})$ three times a day and propranolol $20 \mathrm{mg}$ tablet twice a day for four weeks. Patients were asked to report the frequency, duration and severity of their headaches in designed forms at home. Then at the end of the 2nd and 4th weeks of treatment, patients were followed for clinical efficacy.

Results: In terms of duration, frequency and severity of headaches between the two groups of herbal medicine and placebo, the behavior of the two protocols was changed over time $(p<0.001)$. During the 4 weeks, the time and drug interactions, were significant $(\mathrm{p}<0.001)$. In other words, the pattern of changes to the two protocols over time, was different. Also, at the end of the 4th week, there was a significant difference between the two groups $(\mathrm{p}<0.001)$.

Conclusion: The study findings suggest that the Iranian traditional product combination of Viola odorata flowers, Rosa damascena flowers and Coriandrum sativum fruits may be effective in improving headaches in patients with migraine.
\end{abstract}

\section{Corresponding author:}

Assistant Professor Dr. Haleh Tajadini. Kerman University of Medical Sciences, Kerman, Iran.

Tel: +98.9131972312, Fax: +98.3433122023, Email: dr_haleh@yahoo.com

Received: August 09, 2017, Accepted: September 21, 2017, Published: March 2018

iThenticate screening: September 04, 2017, English editing: January 26, 2018, Quality control: February 10, 2018

This article has been reviewed / commented by four experts

(C) 2018 The Authors. This is an open access article under the terms of the Creative Commons Attribution-NonCommercialNoDerivs License, which permits use and distribution in any medium, provided the original work is properly cited, the use is non-commercial and no modifications or adaptations are made. 
Clinical trial registration: The trial was registered at the Iranian registry of clinical trials (IRCT: www.irct.ir) with registered NO. IRCT 2016110830776N1.

Funding: The authors received no financial support for the research, authorship, and/or publication of this article. Keywords: Migraine; Headache; Viola; Coriandrum; Traditional medicine

\section{Introduction}

Headache disorders are a major public-health problem. They are common and give rise to widespread ill-health (1). In classification of headache, migraine is the second most common after tension headaches (2). Migraine is a disorder that affects more than $10 \%$ of the adult population in the world. In the United States, $14.2 \%$ of adults have migraine or severe headaches (3). Although migraine can begin at any age, the initial attack occurs most commonly during adolescence, by the age of 40 (4) or in the productive period of life (5). Migraine is the chronic periodic headache that is among the ten disabling factors throughout the world (6). Migraine also affects people's quality of life at home, in addition to having a negative impact on work and school. Also, migraine is associated with increased healthcare costs (7). Several prophylactic treatments have been used for decreasing the frequency and severity of migraine attacks. Antiepileptics, three-cyclic antidepressants and beta blockers are medicines that are frequently prescribed (8), but their side effects including constipation (9), tremor, weight gain, somnolence (10), polycystic ovarian and orthostatic hypotension (11), are inevitable. A great number of patients cannot tolerate side effects and some patients mention drug resistance against the prophylactic effects of medicines in long-term use (12). These side effects and drug resistances are the main and inevitable complication in migraine prophylactic treatment (13). The limitations of drug therapy in migraine, can justify the good acceptance of complementary and alternative therapies among patients with this disease (14). Migraine has two common types. Migraine without aura is a clinical syndrome, which is actually a headache with specific characteristics, and migraine with aura, which is mainly associated with neurological transient symptoms include hyperactivity, hypoactivity, depression, cravings for particular foods, repetitive yawning, fatigue and neck stiffness and/or pain, and usually preceded or accompanied by symptoms of the patient (15). According to the World Health Organization (WHO), health systems around the world face increase of chronic diseases and health care costs. Patients and health care providers want improvement of health care services with more emphasize on individual-based care, this includes development of traditional and complementary medicine (16). Complementary medicine, according to the WHO, has a long history in maintaining health and prevention and treatment of diseases, especially chronic diseases. One of the most productive branches of complementary medicine is Iranian traditional medicine, with a ten thousand-year history. Traditional and complementary medicines have always been considered as a valid source for novel treatment findings (17). Headache, like medicine, has a history as long as the existence of human beings, and special approaches have been invented for its relief in each historical period including Iranian traditional medicine (18), for example, use of herbal drugs for the treatment of headache by Iranian physicians goes back to the 6th century. Iranian physicians were well aware of specific therapeutic effects of herbal drugs as analgesics or sedatives and their prophylactic effects in special diseases such as headache. Therefore, they used several herbal drugs for the treatment of headache (19). Included among these medicinal plants that were used for headache which are mentioned in the valid books of Iranian traditional medicine are violet (Viola odorata L. (V. odorata)), rose (Rosa damascena L. (R. damascena)) and coriander (Coriandrum sativum L. (C. sativum)), in isolation $(20,21)$ and in the form of a combination (22-24). Also, several studies have investigated the analgesic, sedative, and pre-anesthetic effects of V. odorata in rats (25, 26), analgesic effects of R. damascene on migraine in human studies and animal studies (27-29), and hypnotic effects of this plant in mice (30), and analgesic effects of C. sativum on migraine in human studies (31) in isolation, oral or topical (25-31). There are also overviews that examine the effects of medicinal plants, which have shown the analgesic effect of these three herbs $(32,33)$. But there is no clinical evidence showing the efficacy of a herbal medicine combination of $V$. odorata, $R$. damascena and $C$. sativum on pain or migraine. This is the first study to be done on such a combination that is derived from authentic texts of traditional medicine. Therefore, we aimed to investigate the effect of an herbal medicine combination of $V$. odorata flowers, $R$. damascene flowers and $C$. sativum fruits on migraine assuming the possibility of decreasing severity, duration and frequency of headaches in a clinical trial.

\section{Material and Methods}

\subsection{Trial design and participants}

This double blind randomized clinical trial (registered NO. IRCT 2016110830776N1, www.irct.ir), was performed on patients with migraine headaches who visited Besat Neurology Clinic NO.4 at Kerman University of Medical Sciences, Kerman, Iran, from September 2016 to march 2017. The diagnosis of migraine based on the International 
Headache Society (IHS) criteria (15), and assessment of the inclusion and exclusion criteria of this study was done by the same and constant neurologist.

\subsection{Selection criteria}

\subsubsection{Inclusion criteria:}

Patients with migraine headache (without aura and with aura) according to the IHS, aged 15-45 years old, patient's awareness toward his/her disease and experiencing at least four migraine attacks in the past 3 months.

2.2.2. Exclusion criteria:

Patient's non-cooperation, decision for pregnancy or having positive pregnancy test, lactation, history of serious medical problems and concurrent treatment (herbal or chemical medicine) such as gastric reflux, diseases of heart, liver and kidney disease, diabetes and depression, and not following proper consumption of prescribed medicine.

\subsection{Research ethics}

The research was approved, by the Ethical Committee of Kerman University of Medical Sciences (IR. KMU.AH.REC.1395.18) in October 2013, based on the latest version of the Helsinki Statement of Ethical Principles. Participation in the study was voluntary. Would-be participants were required to read the informed consent form and sign if they wished to participate in the study. The patients were advised to report any side effect after medication.

\subsection{Interventions}

The intervention group received a traditional medicine product which is a combination of $V$. odorata flower, $R$. damascene flower and $C$. sativum fruits in equal proportions with each other, in $500 \mathrm{mg}$ capsules three times a day, each time, one $500 \mathrm{mg}$ capsule $(1.5 \mathrm{mg}$ /day), and propranolol $20 \mathrm{mg}$ tablet twice a day each time, one tablet, after meals for four weeks. The control group received similar capsules (in regard to color, odor and shape) containing starch powder, three times a day and propranolol $20 \mathrm{mg}$ tablet twice a day after meals similar to the intervention group, for four weeks also.

\subsection{Preparation of Traditional Medicine product Combination of $V$. odorata, $R$. damascena and C. sativum}

First, medicinal plants including viola flower $(V$. odorata $L$.), rose flower $(R$. damascena $L$.) and coriander fruits $(C$. sativum L.) were purchased from the Iran herbal medicine market and were approved by a pharmacognosist in the pharmacy department of Kerman University of Medical Sciences (herbarium number: V. Odorata L. (Kf 1425), R. Damascena L. (Kf 1362), C. Sativum L. (Kf 1524)). Then, qualitative and microbial controls were performed at Barij Essence Company (Kashan, Iran). The medicine was prepared based on the techniques mentioned in Iranian traditional medicine texts. First, herbal medicines were powdered and prepared in the form of 500mg capsules containing viola flower, rose flower and coriander fruit, in equal ratios.

\subsection{Outcomes}

First, baseline and demographic features (sex, age, type of migraine [without aura or with aura], marital status, severity, duration, frequency of headaches) of patients were recorded. Patients were asked to say the average frequency and duration of their headaches in the past 3 months. Then, average severity of headache was evaluated using a visual analog scale (VAS). The VAS is the most common method in which the severity of pain is determined based on behavioral factors, and has ten points. Based on the VAS scoring system, VAS=0: indicated no pain and $\mathrm{VAS}=10$ indicated most severe pain (34). Pain score is entered in a specific predesigned table. Finally, patients were asked to report the frequency, duration and severity of their headaches in designed forms at home. At the end of the 2nd and 4th weeks of treatment, patients were referred to the neurologist to report the mentioned points and evaluation of any side effects of the herbal medicine. All assertions of patients were kept in their own special file.

\subsection{Sample size}

The following formula (35), applicable in longitudinal studies for comparison of two groups, was used in the present study:

$\mathrm{n}=2\left(\mathrm{Z}_{\alpha / 2}+\mathrm{Z}_{\beta}\right)^{2} \sigma^{2}(1+(\mathrm{m}-1) \rho) / \mathrm{md}^{2}$

Where $\alpha=0.05,1-\beta=0.8, \mathrm{~m}=2$ (the number of measurements for each subject) and based on the results of a similar study (35), $\rho=0.05$ (process correlation), $d=2$ (least significant difference to be detected for severity of pain) and $\sigma=4.5$ (process variance) were considered. Finally, sample size for each group was determined as at least 43 patients. 


\subsection{Randomization and blinding}

Randomization was done using foursome blocks in 1:1 ratio for intervention and placebo group, by an epidemiologist. After that, the allocation was done randomly to the groups, by a pharmacist, who cooperated with this study, then all participants were referred to the pharmacist who coded the medications, to receive their drugs. The study was double-blind. It was explained to patients that they were in two different treatment groups, but they were not informed about the nature of the treatments, and none of the assessors or the patients were aware of the allocations. The number of measurements for each patient was twice after the intervention. Allocation concealment was kept until the end of the study. The results were recorded in a specially designed checklist by an assessor. . Figure 1 shows the flowchart of the study.

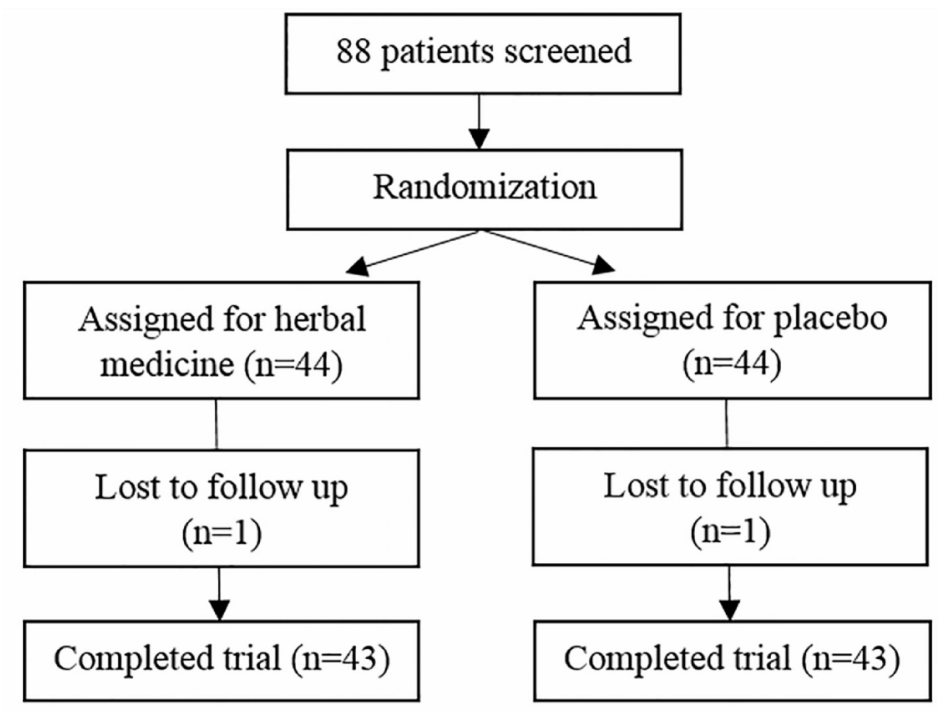

Figure 1. Flowchart of study (Consort diagram)

\subsection{Statistical methods}

All data were analyzed using SPSS software (version 21). Demographic data were presented descriptively as frequencies and means with SDs where appropriate. Chi-square, independent-samples t-test, and repeated measure ANOVA were used to determine the efficacy of herbal medicine versus placebo in migraine attacks. The significance level was determined as 0.05 for two-sided tests.

\section{Results}

In the present study, 88 patients who had migraine headache, were divided into two groups. One of the patients in the intervention group, was not followed because of inappropriate use of prescription drugs, and one of them in the control group was excluded from the study because of positive pregnancy test. Therefore 43 patients in each group were able to finish the study. There was no significant difference between the two groups in terms of age, sex, marital status, and type of migraine (without aura and with aura) (Table 1). There was no significant difference between the two groups of herbal medicine and placebo in the zero week in terms of duration (hour) of headaches $(p=0.594)$. The behavior of the two protocols was changed over time $(p<0.001)$. During the 4 weeks, the time and drug interactions, were significant $(\mathrm{p}<0.001)$. In other words, the pattern of changes to the two protocols over time, was different. Also, at the end of the 4 th week, there was a significant difference between the two groups $(p<0.001)$ (Figure 2). There were no significant differences between the two groups of herbal medicine and placebo in the zero week in terms of frequency (per week) of headaches $(\mathrm{p}=0.944)$. The behavior of the two protocols was changed over time $(p<0.001)$. During the 4 weeks, the time and drug interactions, were significant $(p<0.001)$. In other words, the pattern of changes to the two protocols over time was different. Also, at the end of the 4th week, there was a significant difference between the two groups $(p<0.001)$ (Figure 3$)$. There were no significant differences in the severity (VAS score) of attacks between the two groups of herbal medicine and placebo in the zero week $(\mathrm{p}=0.886)$. The behavior of the two protocols was changed over time $(p<0.001)$. During the 4 weeks, the time and drug interactions, were significant $(\mathrm{p}<0.001)$. In other words, the pattern of changes to the two protocols over time, was 
different. Also, at the end of 4 th week, there was a significant difference between the two groups $(\mathrm{p}<0.001)$ (Figure 4).

Table 1. Demographic Characteristic in the studied groups.

\begin{tabular}{|c|c|c|c|c|}
\hline \multicolumn{2}{|l|}{ Variable } & Intervention & Control & p-value* \\
\hline \multirow[t]{2}{*}{ Gender; n (\%) } & Female & $30(69.0)$ & $32(74.0)$ & \\
\hline & Male & $13(30.0)$ & $11(25.0)$ & \\
\hline \multirow[t]{2}{*}{ Marital status; n (\%) } & Single & $11(25.0)$ & $8(18.0)$ & \multirow[t]{2}{*}{0.43} \\
\hline & Married & $32(74.0)$ & $35(81.0)$ & \\
\hline \multirow[t]{2}{*}{ Type of migraine; n (\%) } & Without aura & $35(81.0)$ & $37(86.0)$ & \multirow[t]{2}{*}{0.56} \\
\hline & With aura & $8(18.0)$ & $6(13.0)$ & \\
\hline Age (year); (Mean $\pm \mathrm{SD})$ & & $32 \pm 5$ & $34 \pm 4$ & 0.093 \\
\hline
\end{tabular}

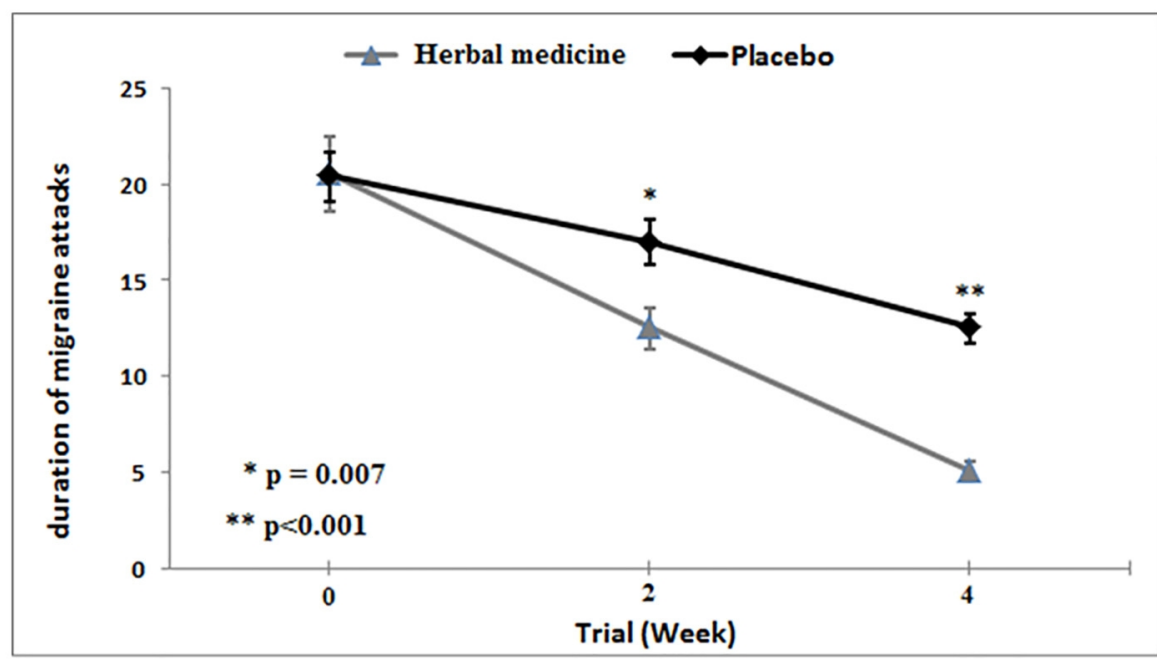

Figure 2. Mean (SE) of duration (hour) in herbal medicine and placebo groups at different time points throughout the study period.

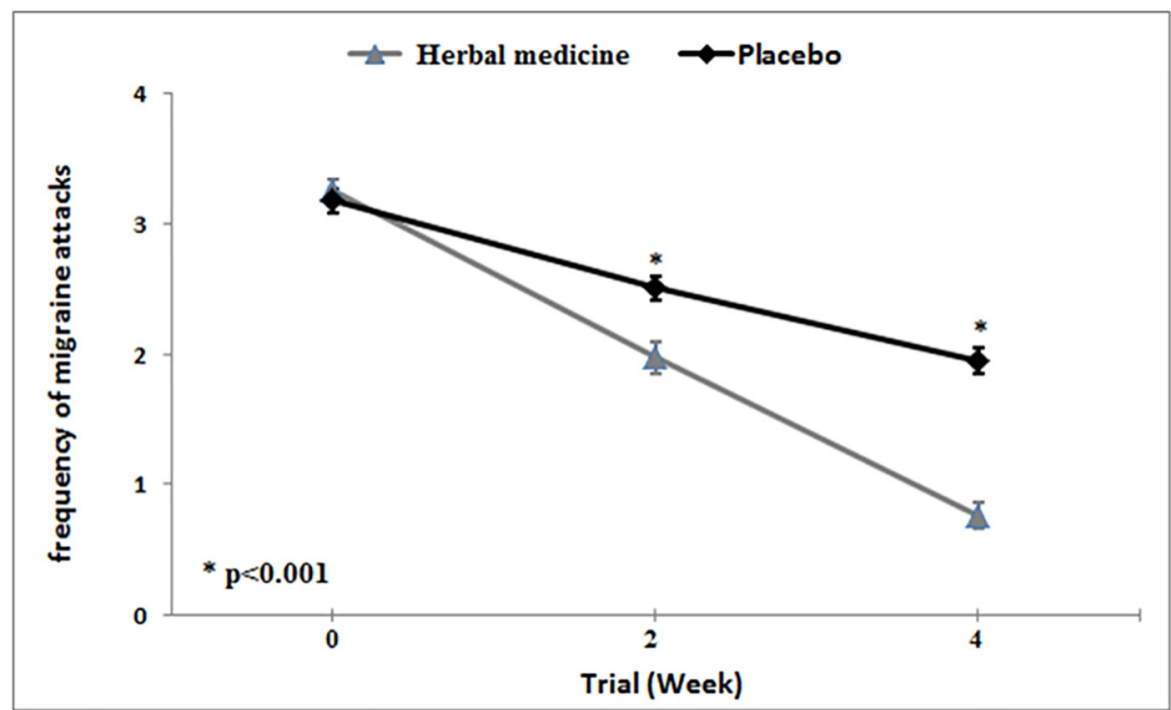

Figure 3. Mean (SE) of frequency (per in a week) in herbal medicine and placebo groups at different time points throughout the study period. 


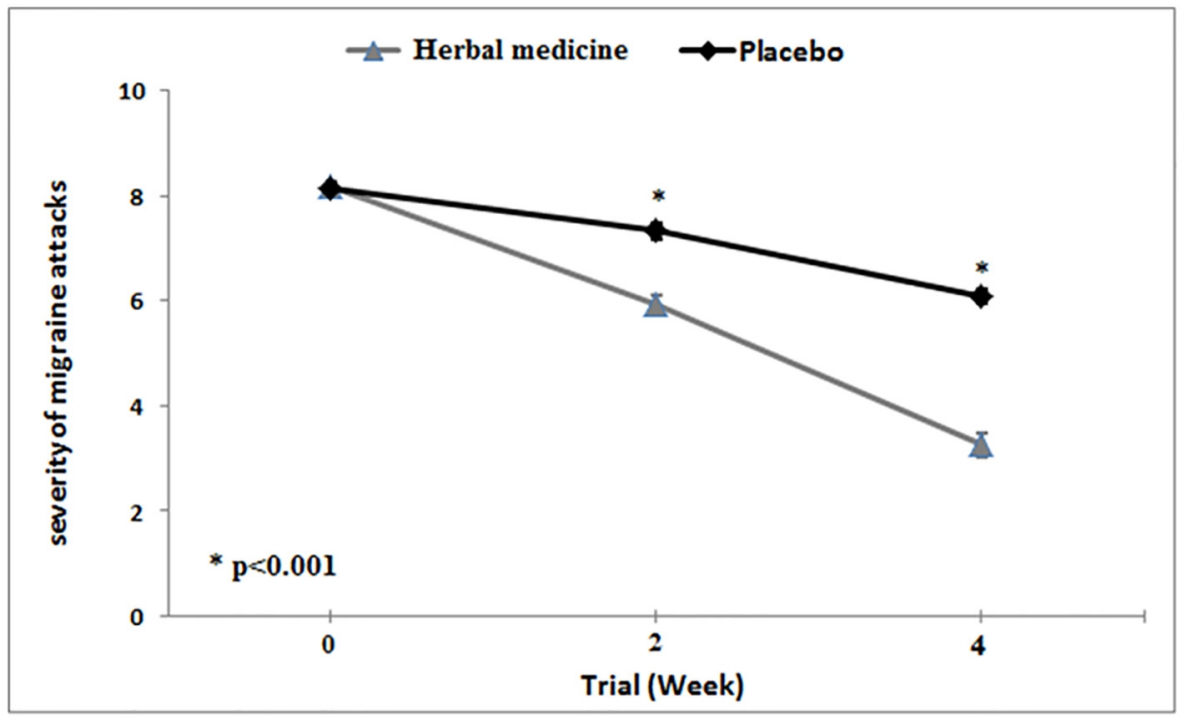

Figure 4. Mean (SE) of severity (VAS score) in herbal medicine and placebo groups at different time points throughout the study period

\section{Discussion}

The obtained findings show that the Iranian traditional medicine combination of $V$. odorata flower, $R$ damascene flower and C. sativum fruit is effective in improving frequency, duration and severity of migraine attacks. This is the first study to investigate the clinical efficacy of this combined herbal medicine in patients with migraine. Hence, we are not able to compare the results we faced here with other studies. With regard to each individual herbal plant, which this herbal product contains, some evidence exists about their analgesic, sedative, anti-inflammatory and hypnotic effects, on headache in human or animal models. Feizabadi et al. studied efficacy of $V$. odorata in the treatment of chronic insomnia in this study, in which violet oil was used. The authors believe that violet oil is an effective medication for insomnia in Iranian traditional medicine. In this study, the effect of violet oil on chronic insomnia was evaluated in pre-test and post-test in 50 patients referred to the Iranian Traditional Medicine Clinic of Mashhad University of Medical Sciences. The treatment consisted of the use of violet oil in the form of an intranasal, two drops in each nostril, each night, before bedtime for one month. Insomnia intensity index (ISI) was completed for all patients before and after one month of treatment. The results indicated that sleep deprivation had decreased scores in patients after one month of use of violet intranasal oil $(\mathrm{p}<0.05)$. In this study, there was no evidence of severe side effects due to the use of violet oil. According to these results, the authors suggest that violet oil can be a tolerable and effective drug for chronic insomnia (36). Vishal Antil et al. performed a study aiming to evaluate, experimentally, the analgesic effect of $n$-hexane, butanolic, methanolic, and aqueous extracts of the aerial part of $V$. odorata at a dose level of 200 and $400 \mathrm{mg} / \mathrm{kg}$, p.o. in rats. In this study, the analgesic effect of aqueous $(p<0.01)$ and methanolic extract $(p<0.05)$ of Viola odorata at a dose of $400 \mathrm{mg} / \mathrm{kg}$, p.o., in the peripheral and central models of pain (tail immersion and hot plate) was significant, but the n-hexane and butanolic extract did not show significant analgesic effects $(\mathrm{P}>0.05)(25)$. Alireza Monadi et al. evaluated the sedative and pre-anesthetic effects of $V$. odorata extract compared with diazepam in rats. Diazepam is a long-acting benzodiazepine. This study mentions that $V$. odorata contains alkaloid, glycoside, saponins, methyl slicylate, mucilage and vitamin $\mathrm{C}$. In this experimental study, $30 \mathrm{Wistar}$ male rats weighing $300 \pm 10 \mathrm{~g}$ and about 3 months old were used. The rats were divided into six groups of five. In order to investigate the effects of sedative and pre-anesthetic violet flower extract in comparison with diazepam, in the first group of mice, $100 \mathrm{mg} / \mathrm{kg}$ of extract, in the second group, $200 \mathrm{mg} / \mathrm{kg}$ of extract, in the third group, $400 \mathrm{mg} / \mathrm{kg}$, in the fourth group, $1.2 \mathrm{mg} / \mathrm{kg}$ diazepam, in the fifth group, $1.2 \mathrm{mg} / \mathrm{kg}$ placebo (dimethyl sulfoxide), was injected intra peritoneal. No drug was given to the sixth group. The results indicated that there was no significant difference between the sedative and pre-anesthetic effects of 100 and 200 $\mathrm{mg} / \mathrm{kg} \mathrm{BW}$ doses of violet flower extract compared to diazepam. But the dose of $400 \mathrm{mg} / \mathrm{kg} \mathrm{BW}$ was a better sedative and had pre-anesthetic effects. The authors of this paper suggest that violet flower extract has better sedative and pre-anesthetic effects than diazepam. But the effects are dose dependent (26). 
Hossein Delavar Kasmaei et al. compared the effects of C. sativum syrup on migraine in a clinical trial with placebo. In this study, 68 patients were equally divided between the intervention and placebo groups. The intervention group received $500 \mathrm{mg}$ of sodium valproate daily with $\mathrm{C}$. sativum syrup three times a day. But, the placebo group received sodium valproate $500 \mathrm{mg}$ tablets daily with placebo syrup three times a day. Both groups were treated for one month. Then, frequency of migraine attacks per week, and the duration and severity of headache attacks were evaluated at weeks 12-3 and 4. They found that the coriander fruit syrup decreased duration, severity and frequency of migraine, in the intervention group $(\mathrm{p}<0.001)$, to be more precise, the mean migraine duration, severity and frequency, in the intervention group, were 5.7 hours, 3.65 units and about $50 \%$ less than the control group, respectively (31). Hassan Rakhshandah et al. studied analgesic effects of the aqueous, ethanolic and chloroformic extracts of $R$. damascene in mice. Mice were treated with intraperitoneal injection of 100,500 and $1000 \mathrm{mg} / \mathrm{kg}$ aqueous, ethanolic and chloroformic extract of roses and their antinociceptive effects were evaluated using hot plate and tail flick methods. The results indicated that the ethanolic extract of roses had stronger effects than morphine, but there was no antinociceptive effect with aqueous and chloroformic extracts (29). Maria Niazi et al. studied efficacy of topical rose ( $R$. damascena) oil for migraine headache in a randomized double-blinded placebocontrolled cross-over trial. Forty patients with migraine headache were randomly assigned to 2 groups of this double-blind, placebo-controlled cross-over trial. The patients were treated for the first 2 consecutive migraine headache attacks by topical $R$. damascena oil or placebo. Then, after one week of washout period, cross-over was done. Pain intensity of the patients' migraine headache was recorded at the beginning and ten sequence time schedules of attacks up to 24 hours. In addition, photophobia, phonophobia, and nausea and/or vomiting (N/V) of the patients were recorded as secondary outcomes. Finally, gathered data were analyzed in a syndrome differentiation manner to assess the effect of $R$. damascena oil on Hot and Cold type migraine headache. They found that mean pain intensity of the patients' migraine headache in the different time points after R. damascene oil or placebo use, was not significantly different. Additionally, regarding mean scores of N/V, photophobia, and phonophobia severity of the patients, no significant differences between the two groups were observed. Finally, applying a syndrome differentiation model, the mean score of migraine headache pain intensity turned out to be significantly lower in patients with "hot" type migraine syndrome at 30, 45, 60, 90, and 120 min after $R$. damascena oil application compared to "cold" types (p-values: $0.001,0.001,<0.001,<0.001$, and 0.02 ; respectively). It is suggested that syndrome differentiation can help in selection of patients who may benefit from the topical $R$. damascena oil in short-term relief of pain intensity in migraine headache. Finally, they mention in this study that, further studies of longer follow-up and larger study population, however, are necessary for more scientifically rigorous judgment on efficacy of $R$. damascena oil for patients with migraine headache (27).

Valiallah Haj Hashemi et al., in an animal model using rats, investigated the antinociceptive and anti-inflammatory effects of hydroalcoholic extract of R. damascena and its essential oil. The extract was administered in doses of 250 , 500 and $1000 \mathrm{mg} / \mathrm{kg}$, and essential oil in doses of 100, 200 and $400 \mu \mathrm{L} / \mathrm{kg}$ orally. To evaluate the analgesic effects, the acetic acid-induced writhing response, formalin-induced paw licking time in the early and late phases and light tail flick test were used in mice. For evaluation of anti-inflammatory effect, carrageenan-induced paw edema, served as a valid animal model in rats, was used. The results showed that the extract significantly reduced the responses stimulated by intraperitoneal injection of acetic acid, and the effect of strong antinociception in both phases of formalin test were shown. Also, the high dose of the extract significantly reduced carrageenan- induced paw edema. Rose essential oil did not have an anti-inflammatory or analgesic effect in any of the prescribed doses (28). Of the possible side effects of $V$. odorata, is nausea, of the $R$. damascene, is decrease in libido and thirst, and of the $C$. sativum, is diarrhea, decrease in libido and forgetfulness (21). One of the limitations of the present study was consumption of this herbal medicine for only one month, that makes it impossible to claim anything about its efficacy in long-term use. Moreover, our patients were not followed-up after the intervention period. Therefore, the results of studying long-term effects with greater sample size can be generalized better. Menstruation of women, which can cause headache or exacerbate headaches, the arbitrary use of herbal medicines or sedative and antiinflammatory chemical drugs, (although patients were asked not to use any herbal or chemical drugs) were other limitations of this study.

\section{Conclusions}

The study findings suggest that the Iranian traditional product combination of $V$. odorata, $R$. damascena and $C$. sativum may be effective in improving headaches in patients with migraine. Four weeks administration of this herbal medicine (V. odorata, $R$. damascena and $C$. sativum) associated with improved frequency, duration and severity of headache attacks without any serious side effects in patients with migraine. We recommend further studies on 
human subjects with longer follow-ups and comparison to chemical drugs currently used for migraine, to find any advantage or disadvantage of this herbal medicine.

\section{Clinical trial registration:}

The trial was registered at the Iranian registry of clinical trials (IRCT: www.irct.ir) with registered NO. IRCT $2016110830776 \mathrm{~N} 1$.

\section{Funding:}

The authors received no financial support for the research, authorship, and/or publication of this article.

\section{Acknowledgments:}

This article is based on part of a PhD thesis, at the School of Iranian Traditional Medicine, Kerman University of Medical Sciences, Kerman, Iran.

\section{Conflict of Interest:}

There is no conflict of interest to be declared.

\section{Authors' contributions:}

All authors contributed to this project and article equally. All authors read and approved the final manuscript.

\section{References:}

1) Schramm S, Uluduz D, Gouveia RG, Jensen R, Siva A, Uygunoglu U, et al. Headache service quality: evaluation of quality indicators in 14 specialist-care centres. J Headache Pain. 2016; 17(1): 111. doi: 10.1186/s10194-016-0707-9. PMID: 27933580, PMCID: PMC5145818.

2) Huguet A, Tougas ME, Hayden J, McGrath PJ, Chambers CT, Stinson JN, et al. Systematic Review of Childhood and Adolescent Risk and Prognostic Factors for Recurrent Headaches. J Pain. 2016; 17(8): 85573. doi: 10.1016/j.jpain.2016.03.010. PMID: 27102894.

3) Lee J, Bhowmick A, Wachholtz A. Does complementary and alternative medicine (CAM) use reduce negative life impact of headaches for chronic migraineurs? A national survey. Springerplus. 2016; 5(1): 1006. doi: 10.1186/s40064-016-2362-7. PMID: 27398280, PMCID: PMC4937006.

4) Darof RB, Fenichel GM, Jancovic J, Mazziotta JC. Bradley's Neurology in Clinical Practice. 2012; 2: 1715.

5) Wang SJ, Chen PK, Fuh JL. Comorbidities of Migraine. Front Neurol. 2010; 1: 16. doi: 10.3389/fneur.2010.00016. PMID: 21188255, PMCID: PMC3008936.

6) Cady RK, Munjal S, Cady RJ, Manley HR, Brand-Schieber E. Randomized, double-blind, crossover study comparing DFN-11 injection (3 mg subcutaneous sumatriptan) with $6 \mathrm{mg}$ subcutaneous sumatriptan for the treatment of rapidly-escalating attacks of episodic migraine. J Headache Pain. 2017; 18(1): 17. doi: 10.1186/s10194-016-0717-7. PMID: 28176235, PMCID: PMC5296269.

7) Holroyd KA, Cottrell CK, O’Donnell FJ, Cordingley GE, Drew GB, Carlson BW, et al. Effect of preventive ( $\beta$ blocker) treatment, behavioural migraine management, or their combination on outcomes of optimised acute treatment in frequent migraine: randomised controlled trial. BMJ. 2010; 341: c4871. doi: 10.1136/bmj.c4871.

8) Garza I, Swanson JW, Cheshire WP, Boes CJ, Capobianco DJ, Vargas BB, et al. Headache and other craniofacial pain. Bradley neurology in clinical practice. Philadelphia: Elsevier. 2012; 2: $1703-44$. doi: 10.1016/B978-1-4377-0434-1.00094-3.

9) Uher R, Farmer A, Henigsberg N, Rietschel M, Mors O, Maier W, et al. Adverse reactions to antidepressants. Br J Psychiatry. 2009; 195(3): 202-10. doi: 10.1192/bjp.bp.108.061960. PMID: 19721108.

10) Ramakrishnappa SK, Belhekar MN. Serum drug level-related sodium valproate-induced hair loss. Indian J Pharmacol. 2013; 45(2): 187-8. doi: 10.4103/0253-7613.108315. PMID: 23716898, PMCID: PMC3660934.

11) Miller S. The acute and preventative treatment of episodic migraine. Ann Indian Acad Neurol. 2012; 15(Suppl 1): S33-9. doi: 10.4103/0972-2327.99998. PMID: 23024562, PMCID: PMC3444218.

12) Adams J, Barbery G, Lui CW. Complementary and alternative medicine use for headache and migraine: a critical review of the literature. Headache. 2013; 53(3): 459-73. doi: 10.1111/j.1526-4610.2012.02271.x. PMID: 23078346. 
13) Tajmirriahi M, Sohelipour M, Basiri K, Shaygannejad V, Ghorbani A, Saadatnia M. The effects of sodium valproate with fish oil supplementation or alone in migraine prevention: A randomized single-blind clinical trial. Iran J Neurol. 2012; 11(1): 21-4. PMID: 24250854, PMCID: PMC3829229.

14) Wells RE, Bertisch SM, Buettne C, Phillip RS, McCarthy EP. Complementary and alternative medicine use among adults with migraines/severe headaches. Headache. 2011; 51(7): 1087-97. doi: 10.1111/j.15264610.2011.01917.x. PMID: 21649654, PMCID: PMC3627391.

15) Headache Classification Committee of the International Headache Society (IHS). The International Classification of Headache Disorders, 3rd edition (beta version). Cephalalgia. 2013; 33(9): 629-808. doi: 10.1177/0333102413485658. PMID: 23771276.

16) Burton A, Falkenberg T, Smith M, Zhang Q. WHO traditional medicine strategy 2014-2023. Available from: http://www.who.int/medicines/publications/traditional/trm_strategy14_23/en/.

17) Zargaran A, Borhani-Haghighi A, Faridi P, Daneshamouz S, Mohagheghzadeh A. A review on the management of migraine in the Avicenna's Canon of Medicine. Neurol Sci. 2016; 37(3): 471-8. doi: 10.1007/s10072-016-2498-9. PMID: 26861565.

18) Zarshenas MM, Petramfar P, Firoozabadi A, Moein MR, Mohagheghzadeh A. Types of headache and those remedies in traditional persian medicine. Pharmacogn Rev. 2013; 7(13): 17-26. doi: 10.4103/09737847.112835. PMID: 23922452, PMCID: PMC3731875.

19) Gorji A. Pharmacological treatment of headache using traditional Persian medicine. Trends Pharmacol Sci. 2003; 24(7): 331-4. doi: 10.1016/S0165-6147(03)00164-0. PMID: 12871664.

20) Avicenna. The Cannon of Medicine. Tehran, Iran: Sorush Publication; 1983.

21) Aghili Khorasani MH. Tehran: Tehran University of Medical Sciences; 2013.

22) Chashti H. Exir-e-Azam. Tehran: Thran University of Medical Sciences, Institute for Islamic and Complementary Medicine. 2008.

23) A’azam khan M. Gharabadin-e azam va akmal. Tehran: AL-Maei; 2014: 369.

24) Karim khan kermani H. Daqaeq- al- AlajTehran: Chogan; 2016: 233.

25) Antil V, Kumar P, Kannappan N, Diwan A, Saini P, Singh S. Evaluation of the analgesic activity of Viola odorata aerial parts in rats. Journal of Natural Pharmaceuticals. 2011; 2(1): 24-7.

26) Monadi A, Rezaie A. Evaluation of sedative and pre-anesthetic effects of Viola odorata Linn. Extract compared with diazepam in rats. Bull Env Pharmacol Life Sci. 2013; 2(7): 125-31.

27) Niazi M, Hashempur MH, Taghizadeh M, Heydari M, Shariat A. Efficacy of topical Rose (Rosa damascena Mill.) oil for migraine headache: A randomized double-blinded placebo-controlled cross-over trial. Complement Ther Med. 2017; 34: 35-41. doi: 10.1016/j.ctim.2017.07.009. PMID: 28917373.

28) Hajhashemi V, Ghannadi A, Hajiloo M. Analgesic and anti-inflammatory effects of Rosa damascena hydroalcoholic extract and its essential oil in animal models. Iran J Pharm Res 2010; 9(2): 163-8. PMID: 24363723, PMCID: PMC3862064.

29) Rakhshandeh H, Vahdati-Mashhadian N, Dolati K, Hosseini M. Antinociceptive effect of Rosa damascena in Mice. J Biol Sci. 2008; 8: 176-80. doi: 10.3923/jbs.2008.176.180.

30) Rakhshandah H, Hosseini M, Dolati K. Hypnotic Effect of Rosa damascena in Mice. Iranian Journal of Pharmaceutical Research. 2004; 3(3): 181-5.

31) Delavar Kasmaei H, Ghorbanifar Z, Zayeri F, Minaei B, Kamali SH, Rezaeizadeh H, et al. Effects of Coriandrum sativum Syrup on Migraine: A Randomized, Triple-Blind, Placebo-Controlled Trial. Iran Red Crescent Med J. 2016; 18(1): e20759. doi: 10.5812/ircmj.20759. PMID: 26889386, PMCID: PMC4752800.

32) Mahdizadeh S, Khaleghi Ghadiri M, Gorji A. Avicenna's Canon of Medicine: a review of analgesics and anti-inflammatory substances. Avicenna J Phytomed. 2015; 5(3): 182-202. PMID: 26101752, PMCID: PMC4469963.

33) Hamedi A, Zarshenas MM, Sohrabpour M, Zargaran A. Herbal medicinal oils in traditional Persian medicine. Pharm Biol. 2013; 51(9): 1208-18. doi: 10.3109/13880209.2013.777462. PMID: 23746335.

34) Bijur PE, Silver W, Gallagher EJ. Reliability of the Visual Analog Scale for Measurement of Acute Pain. Acad Emerg Med. 2001; 8(12): 1153-7. doi: 10.1111/j.1553-2712.2001.tb01132.x. PMID: 11733293.

35) Fallah R, Divanizadeh MS, Karimi M, Mirouliaei M, Shamszadeh A. Topiramate and propranolol for prophylaxis of migraine. Indian J Pediatr. 2013; 80(11): 920-4. doi: 10.1007/s12098-013-0976-0. PMID: 23412986.

36) Feyzabadi Z, Jafari F, Kamali SH, Ashayeri H, Badiee Aval S, Esfahani MM, et al. Efficacy of Viola odorata in Treatment of Chronic Insomnia. Iran Red Crescent Med J. 2014; 16(12): e17511. doi: 10.5812/ircmj.17511. PMID: 25763239, PMCID: PMC4341349. 